\title{
Flexión compuesta esviada en secciones de hormigón armado doblemente simétricas. Dirección de capacidad máxima
}

\author{
Biaxial bending in double simmetrical reinforced concrete sections. \\ Major capacity direction
}

M. Martínez $^{(*)}$, R. Lanti ${ }^{(*)}$, E. Echeverria ${ }^{(*)}$

RESUMEN

El uso de modelos constitutivos simplificados para caracterizar el comportamiento del hormigón, (parábola rectángulo, bloque rectangular), conduce a que la capacidad de agotamiento de una sección doblemente simétrica solicitada por un axil excéntrico se encuentre en la dirección de simetría principal. Si se evalúa la capacidad de la sección empleando un modelo hiperbólico para la descripción del hormigón se obtiene que para axiles elevados, la dirección de resistencia máxima presenta un cierto desvío respecto a la dirección de simetría principal tal como se obtiene con el uso de modelos simplificados. En este trabajo se determinan y comparan una serie de diagramas de interacción en flexión compuesta esviada $\left(M_{x}, M_{\nu}\right)$ de dos secciones con cuantías y axiles crecientes y dos modelos constitutivos de hormigón (parábola rectángulo e hiperbólico) a fin de determinar la dirección en la que se encuentra la resistencia máxima de las secciones en los casos analizados.

Palabras clave: diagrama de interacción, flexión esviada, soportes de hormigón.

\section{ABSTRACT}

The use of simplified stress-strain relationship to describe the behavior of the concrete in compression (rectangular parabola, rectangular block), leads to find that the maximum capacity of a double symmetric reinforced concrete section under axial load an biaxial bending in the main symmetrical axis of the section. For the same section, the use of hyperbolic stress-strain relationship to describe the concrete behavior is found that the maximum capacity of the section under elevated compression level and biaxial bending does not lay in the symmetry axis direction but in an arbitrary one. A collection of interaction diagrams, biaxial bending an axial load, $\left(\mathrm{M}_{\mathrm{x}}, \mathrm{M}_{\mathrm{y}}\right)$ of two sections with different amount of steel and two different axial loads are calculated in this research for two different stress-strain relationship for concrete (rectangular parabola, and hyperbolic), in order to find the direction of maximum resistance of the section under axial load and biaxial bending.

Keywords: interaction diagrams, skew bending, reinforced concrete columns

(*) Universidad de Alcalá de Henares (España).

Persona de contacto/Corresponding author: martinezmartinezmonica@gmail.com (M. Martinez).

ORCID: https://orcid.org/oooo-0003-4544-9517 (M. Martínez); https://orcid.org/oooo-00o2-6406-3954 (R. Lanti); https://orcid.org/oooo-0oo2-6826-5756 (E. Echeverria).

Cómo citar este artículo/Citation: Martínez, M.; Lanti, R.; Echeverria, E. (2019). Flexión compuesta esviada en secciones de hormigón armado doblemente simétricas. Dirección de capacidad máxima. Informes de la Construcción, 71(554): e285. https://doi. org/10.3989/ic.59325

Copyright: (C) 2019 CSIC. Este es un artículo de acceso abierto distribuido bajo los términos de la licencia de uso y distribución Creative Commons Reconocimiento 4.o Internacional (CC BY 4.0). 


\section{INTRODUCCIÓN}

La dificultad numérica que supone llevar a cabo la integración exacta de las tensiones de hormigón y acero en una sección trasversal de hormigón armado solicitada por un axil con excentricidades según los dos ejes de simetría, ha motivado que los códigos reguladores hayan aceptado modelos simplificados para caracterizar el comportamiento tensión deformación del hormigón en compresión. La EHE-o8 (1) permite el empleo de un diagrama parábola rectángulo (figura 1a) en el que la tensión de agotamiento $\left(f_{c}\right)$ se alcanza para un valor de deformación $\varepsilon_{0}=0.002$, y ésta tensión permanece constante hasta alcanzar el valor de deformación de agotamiento $\varepsilon_{u}$. La Instrucción permite igualmente el empleo de un modelo constitutivo rectangular en el que se reduce la profundidad real de la fibra neutra y se otorga a la zona comprimida el valor de la tensión de agotamiento (hipótesis planteada y desarrollada por Withney (2) en 1956).

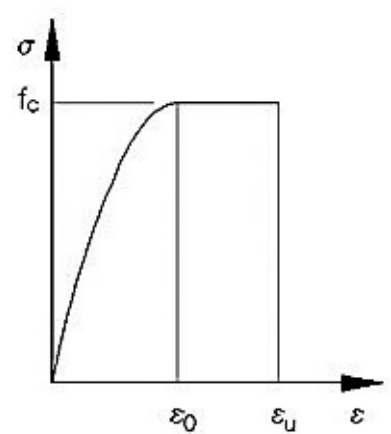

(a)

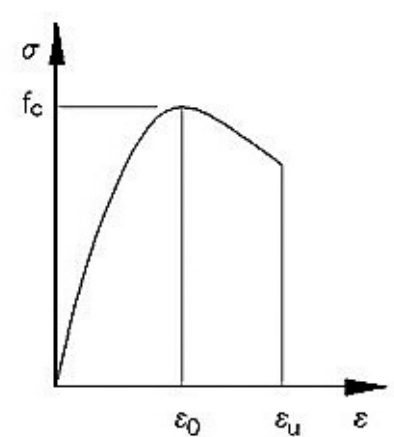

(b)
Figura 1. (a) Diagrama tensión deformación para un modelo parábola rectángulo (b) Diagrama tensión deformación para un modelo hiperbólico.

Un modelo constitutivo para el hormigón más realista es el que se muestra en la figura $1 \mathrm{~b}$, en la línea de las investigaciones llevadas a cabo por Hognestad (3), Kent (4), Sargin (5) entre otros, en el cual la tensión correspondiente a la deformación de agotamiento no es la máxima tensión a compresión del hormigón. En estos modelos se observa una caída significativa de la tensión respecto a la tensión máxima para deformaciones próximas a las de agotamiento.

La elección del modelo constitutivo para la determinación de la capacidad de la sección, condiciona el resultado final de manera determinante. Así, si se calculan diagramas de interacción $\left(M_{x}, M_{y}\right)$ de una sección con dos ejes de simetría y un nivel de axil constante con modelos constitutivos de hormigón simplificados (figura 1a), parece verificarse la propiedad postulada por Morán (6) respecto a la convexidad de dichos diagramas. En estas situaciones, el valor de resistencia máxima se alcanza según la dirección del eje principal de simetría de la sección (eje $x$ ), que así mismo es el eje principal de inercia.

Sin embargo se observa que en situaciones de axiles elevados y modelos constitutivos hiperbólicos (figura 1b) en los que la tensión de agotamiento se alcanza para deformaciones alejadas de la deformación de rotura, y en los que existe una reducción de tensión frente a la tensión característica en dicha deformación, la capacidad máxima a flexión compuesta no tiene por qué situarse en la dirección que marca el eje de simetría principal de la sección (aparente eje principal de inercia), sino que presenta un cierto desvío respecto de éste. Esto implica que en estas circunstancias los diagramas de interacción pueden presentar concavidades en las zonas próximas a las direcciones que marcan los ejes de simetría de la sección $\left(0^{\circ}, 90^{\circ}\right)$

A pesar de que las diferencias observadas se producen para axiles elevados y estos deben de evitarse en fase de diseño, (se reduce la ductilidad de la sección, y pueden aparecer posibles efectos de inestabilidad a nivel de estructura), los diagramas de interacción publicados en los que se alcanzan estos niveles de compresión, así como los modelos clásicos de dimensionamiento basados en la obtención de una excentricidad única equivalente, no contemplan la reducción de capacidad de la sección según las direcciones de los ejes de simetría $\left(0^{\circ}, 90^{\circ}\right)$ por el empleo de modelos hiperbólicos y otorgan a estas direcciones las direcciones de valores extremos de la sección. En el presente trabajo se analiza esta cuestión y se evalúan las implicaciones que puede tener en el dimensionado de secciones de hormigón armado en compresión o flexión compuesta esviada. Igualmente se investiga el giro existente entre el eje de simetría principal de una sección y el eje principal de inercia en agotamiento.

\section{USO DE MODELOS CONSTITUTIVOS SIMPLIFICADOS PARA LA CARACTERIZACIÓN DEL HORMIGÓN EN COMPRESIÓN}

En el objetivo de llevar a cabo el dimensionado y comprobación de secciones de hormigón armado sometidas a un estado de solicitación normales arbitrario (compresión o flexión compuesta esviada), una técnica aceptada y ampliamente utilizada es la sustitución de las dos excentricidades de diseño referidas a dos ejes de referencia de la sección por una única excentricidad situada sobre el eje principal de la sección que conduzca a un estado equivalente de solicitación exterior al del estado de solicitación original. Abordar el dimensionado en compresión o flexión compuesta recta es desde un punto de vista numérico más simple que el problema general de un axil de compresión solicitando una sección con dos excentricidades puesto que, en este caso, el giro del eje neutro es conocido y coincide con la dirección perpendicular a la dirección de la excentricidad.

En esta línea en 1963 Pannell (7) establece un modelo geométrico para la determinación de diagramas de interacción de secciones cuadradas y distribución homogénea de armadura en las cuatro caras (sección con doble simetría). Para ello parte de dos hipótesis fundamentales, la primera de las cuales se demostrará no necesariamente cierta:

- Los valores máximos de capacidad se encuentra en los planos de simetría de la sección, direcciones principales $\left(0^{\circ}, 90^{\circ}\right)$.

- El valor mínimo de capacidad se encuentra en el plano de la diagonal de la sección, bisectriz de las direcciones principales $\left(45^{\circ}\right)$.

El método establece los valores de agotamiento $\left(M_{x}, M_{y}\right)$ para cualquier dirección de excentricidad mediante interpolación geométrica entre los valores obtenidos para los planos de simetría y diagonal de la sección (figura 2). 


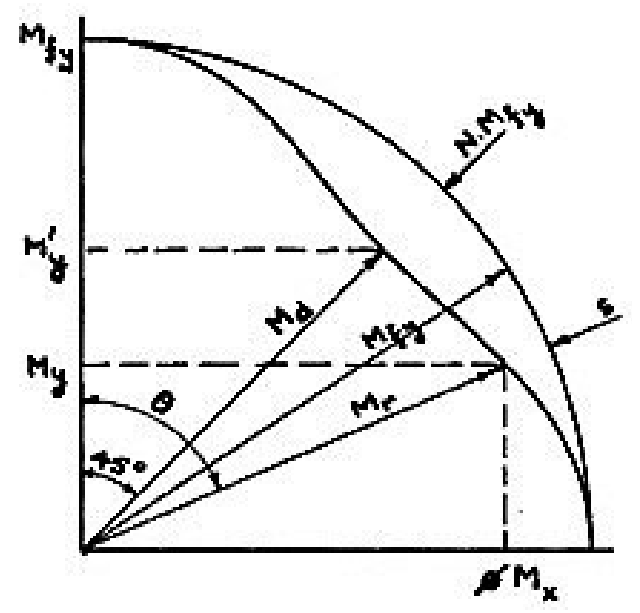

Figura 2. Pannell (1963).

El método A definido por Bresler (8) infiere de manera lineal el axil de agotamiento que solicita una sección según una dirección de cualquiera $\left(P_{i}\right)$. Para ello parte de la necesidad de determinar el axil que agota la sección para cada una de las componentes de la excentricidad de diseño $\left(P_{x}, P_{y}\right)$, así como el axil que agota la sección en compresión centrada $\left(P_{\mathrm{o}}\right)$. Este modelo se puede expresar según la ecuación [1]:

$$
\frac{1}{P_{i}}=\frac{1}{P_{x}}+\frac{1}{P_{y}}-\frac{1}{P_{0}}
$$

Los comentarios de la ACI-318 (9) recogen este modelo y lo presentan como estrategia de cálculo válida de secciones sometidas a estados de compresión o flexión compuesta esviada.

Otros autores publican diagramas adimensionales de interacción para el dimensionamiento y comprobación de secciones sometidas a estados de flexión compuesta esviada para diversos axiles, cuantías y distribución de armadura, entre ellos Parme (10), Weber (11), Row y Paulay (12), o Grasser (13), (figura 3).
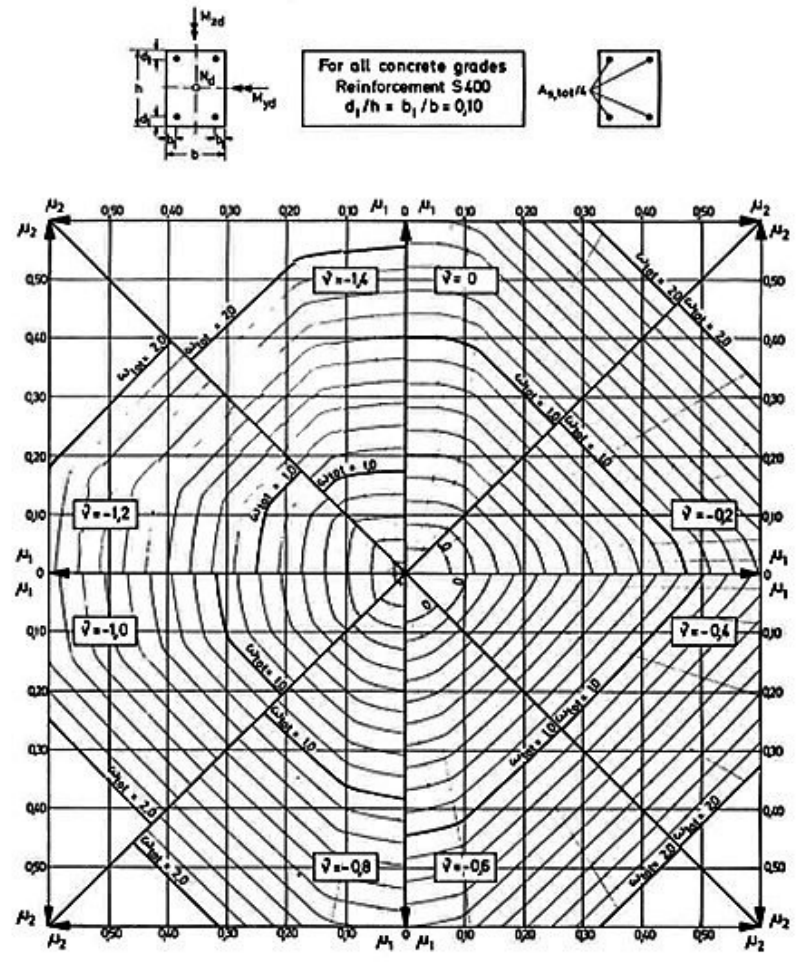

Figura 3. Grasser (1981).
Todos estos modelos llevan a cabo la integración de las tensiones en el bloque comprimido haciendo uso de modelos simplificados para la caracterización del hormigón (parábola rectángulo, bloque rectangular), y esta simplificación implica la reducción de la profundidad de la fibra neutra, lo cual provoca que se eleve el centro de gravedad de la resultante del hormigón, aumentando el brazo mecánico y alcanzándose, en determinadas circunstancias valores de agotamiento superiores a los reales para las direcciones próximas a las de simetría de la sección.

Modelos más recientes optan por la búsqueda de la solución exacta mediante métodos numéricos o bien, tratan de alcanzar el dimensionamiento estricto que conduzca a mínimo coste de acero (problemas de optimización). Estos modelos contemplan el uso de ecuaciones constitutivas arbitrarias y parametrizables, y salvo el trabajo desarrollado por Papanikolau (14), soslayan la debilidad de los modelos de diagramas de interacción clásicos sin poner explícitamente de manifiesto el hecho principal desarrollado en el presente trabajo. En esta línea se encuentran autores como Lejano (15), Gil-Martín (16), Leite (17), o Sanchez-Olivares (18).

\section{DIRECCIÓN DE MÁXIMA RESISTENCIA DE UNA SECCIÓN DE HORMIGÓN ARMADO CON DOBLE SIMETRÍA}

Llevar a cabo la determinación de un diagrama de interacción de agotamiento en el que se representen las componentes del momento último referidos a los dos ejes de simetría de la sección conlleva la necesidad de establecer para cada rotación posible del eje neutro $\left(0^{\circ}-90^{\circ}\right.$ si la sección posee dos ejes de simetría), un plano de deformación cuya traza será el eje neutro postulado. La curvatura de la sección en rotura ha de ser determinada mediante la imposición de la deformación de agotamiento de una de las fibras características de la sección. Según la instrucción EHE-o8 y en consecuencia con el artículo 42.1.3, la sección alcanzará el agotamiento por compresión de la fibra extrema de hormigón comprimido $\left(\varepsilon_{c o}, \varepsilon_{c u}\right)$, o bien por alargamiento excesivo del acero en tracción $\left(\varepsilon_{y}\right)$. Para cada rumbo elegido de eje neutro es posible afirmar que existe un solo plano que agota la sección.

Para un plano de agotamiento cualquiera es posible establecer las ecuaciones de equilibrio [2], [3], [4] y referirlas a un sistema de coordenadas arbitrario. En secciones con dos ejes de simetría es habitual adoptar estos ejes como sistema de referencia.

$$
\begin{gathered}
P=\int_{A} \sigma_{c} d A+\sum_{j=1}^{m} A_{s, j} \sigma_{s, j} \\
M_{x}=\int_{A} \sigma_{c} y d A+\sum_{j=1}^{m} A_{s, j} \sigma_{s, j} y_{s, j} \\
M_{y}=\int_{A} \sigma_{c} x d A+\sum_{j=1}^{m} A_{s, j} \sigma_{s, j} x_{s, j}
\end{gathered}
$$

La elección del modelo constitutivo para el hormigón en la resolución de las ecuaciones de equilibrio [2], [3], [4], condiciona el valor de los esfuerzos de agotamiento $\left(P, M_{x}, M_{y}\right)$ que se logran. De hecho, la asunción de un modelo parábolarectángulo frente a un modelo hiperbólico conduce como se demuestra en este trabajo a que en situaciones donde hubiera sido esperable hallar el valor máximo de capacidad resistente 
según el eje de simetría principal (eje x), este valor extremo se halle para otra dirección. Se analiza y expone en este trabajo esta cuestión, y se lleva a cabo una simulación numérica en la que se comparan para dos secciones doblemente simétricas los diagramas de interacción resultantes del empleo de dos modelos constitutivos distintos: parábola-rectángulo e hiperbólico. Para ello se determinan un total de 24 diagramas de interacción en flexión compuesta esviada. Cada uno de estos diagramas se determina para dos modelos constitutivos de hormigón diferentes y mostrados en la figura 4. La curva tensión deformación mostrada en la figura $4 \mathrm{~b}$ corresponde a la ecuación empleada por Farah y Huggins (19), y descrita en forma polinómica según la expresión [5]. La figura 4a corresponde a la curva de la figura $4 \mathrm{~b}$ en la que se ha establecido un valor constante de tensión (valor de agotamiento) desde el punto en el que se alcanza la deformación $\varepsilon_{0}=0.002$, hasta la deformación en la que se ha considerado se logra el agotamiento $\varepsilon_{c u}=0.004$.

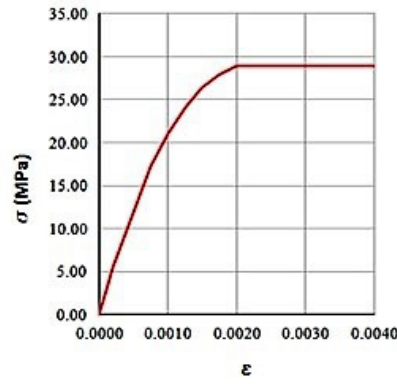

(a)

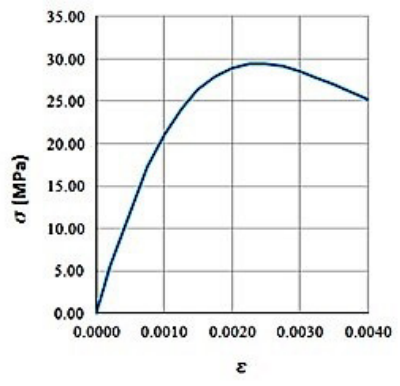

(b)
Figura 4. Farah y Huggins (1969).

$$
\sigma_{c}=f_{c^{\prime}}\left[k_{1} \varepsilon+k_{2} \varepsilon^{2}+k_{3} \varepsilon^{3}+k_{4} \varepsilon^{4}\right]
$$

Donde las constantes denotadas por toman los siguientes valores:

$$
\begin{gathered}
k_{1}=0.985 \cdot 10^{3} \\
k_{2}=-0.312 \cdot 10^{6} \\
k_{3}=0.306 \cdot 10^{8} \\
k_{4}=-0.257 \cdot 10^{9}
\end{gathered}
$$

La curva tensión deformación empleada para la caracterización del acero se ha tomado igualmente de Farah y Huggins, (figura 5) así como la expresión polinómica [6] que la describe de manera continua para todo el rango de deformaciones.

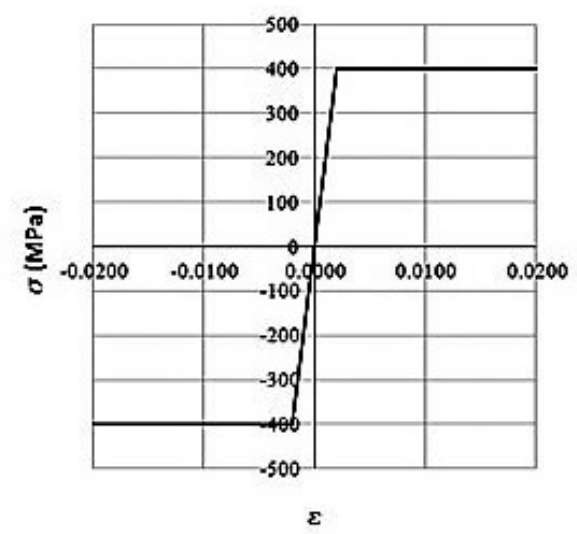

Figura 5. Farah y Huggins (1969).

$$
\sigma_{s}=\frac{f_{y}}{2}\left(\sqrt{\left(\frac{\varepsilon}{\varepsilon_{y}}+1\right)^{2}}-\sqrt{\left(\frac{\varepsilon}{\varepsilon_{y}}-1\right)^{2}}\right)
$$

Para este estudio se han escogido los siguientes valores característicos de hormigón y acero:

$$
\begin{gathered}
f_{c}=30 \mathrm{MPa} \\
f_{y}=400 \mathrm{MPa}
\end{gathered}
$$

Los diagramas calculados se corresponden a dos escuadrías diferentes de sección transversal cuyas relaciones entre sus lados son $\frac{h}{b}=1, y \frac{h}{b}=2$. Para cada una de estas secciones se analizan tres cuantías de armadura longitudinal definidas según la expresión [7], de valores $\omega_{1}=0.30, \omega_{2}=0.40, \omega_{3}=0.50$, y dos axiles de compresión definidos según la expresión [8], de valores $v_{1}=0.85, v_{2}=0.95$. En todos los casos se ha considerado un recubrimiento mecánico de valor $r=0.10 b$. Se emplean los dos modelos constitutivos de hormigón descritos en la figuras $4 \mathrm{a}$ y $4 \mathrm{~b}$.

$$
\begin{gathered}
\omega=\frac{A_{s} f_{y}}{b h f_{c}} \\
v=\frac{P}{b h f_{c}}
\end{gathered}
$$

Los esfuerzos reducidos $\mu_{x}, \mu_{y}$ representados en los diagramas se definen según las expresiones [9] y [10] respectivamente.

$$
\begin{aligned}
& \mu_{x}=\frac{M_{x}}{b h^{2} f_{c}} \\
& \mu_{y}=\frac{M_{y}}{h b^{2} f_{c}}
\end{aligned}
$$

La armadura se ha supuesto distribuida en el perímetro de la sección, y con objeto de poder llevar a cabo la integración de las tensiones se han considerado un total de 36 elementos de igual superficie. Autores como Row y Paulay emplean este tipo de armado en sus estudios, y en este trabajo se ha tratado de dotar al análisis y a las conclusiones extraídas de la máxima generalidad y abstracción, alejándose de configuraciones concretas de armaduras convencionales. Para la simulación de la zona de hormigón se ha dividido la sección en un total de 625 elementos de igual superficie, distribuidos según una matriz de 25x25. Con objeto de validar el análisis presentado en este trabajo se ha realizado la determinación de los diagramas para cuatro casos distintos de tamaño de elemento con el que realizar la integración en el bloque comprimido. Así, se han analizado los casos de 50x50, 100x100 y 200x200, (2500, 10000, 40000 elementos respectivamente), no hallándose diferencias entre los resultados de estos caso y el resultado del caso expuesto en este estudio. La similitud de los resultados hallados entre las diferentes mallas de elementos de hormigón se justifica debido a que este trabajo mide la diferencia de capacidad entre el momento resistente máximo (según la dirección real de inercia máxima en agotamiento) frente al momento resistente para la dirección principal de simetría, y la posible pérdida de precisión por la reducción del número de elementos ( 625 frente a 40000) ocurre para todos los diagramas calculados, y para los dos modelos constitutivos. Así, y con independencia de que los valores de los 
momentos resistentes pueden verse modificados en función del número de elementos, las diferencias relativas entre ellos se conservan, y conducen a soluciones equivalentes.

Para cada curva de interacción determinada y representada en la figura 6 se han calculado un total de 181 puntos correspondientes a un espectro de giros del eje neutro que varía desde $0^{\circ}-90^{\circ}$. Esto equivale a la obtención de una serie de planos de agotamiento cuyas trazas (fibras neutras) en dos puntos consecutivos difieren $0.50^{\circ}$.

Es posible expresar las ecuaciones [2], [3], y [4] en términos de sumatorios de resultantes de cada elemento definido (hormigón y acero) en la sección transversal según las expresiones [11], [12], y [13]:

$$
\begin{gathered}
P=\sum_{i=1}^{n} A_{c, i} \sigma_{c, i}+\sum_{j=1}^{m} A_{s, j} \sigma_{s, j} \\
M_{x}=\sum_{i=1}^{n} A_{c, i} \sigma_{c, i} y_{c, i}+\sum_{j=1}^{m} A_{s, j} \sigma_{s, j} y_{s, j} \\
M_{y}=\sum_{i=1}^{n} A_{c, i} \sigma_{c, i} x_{c, i}+\sum_{j=1}^{m} A_{s, j} \sigma_{s, j} x_{s, j}
\end{gathered}
$$

Para evitar dotar de una capacidad no real a la sección, la tensión de cada elemento de acero se ha modificado para simular el área de hormigón desplazada reduciendo su valor según la expresión [14], para los elementos de acero comprimidos.

$\sigma_{s, i}=\frac{f_{y}}{2}\left(\sqrt{\left(\frac{\varepsilon}{\varepsilon_{y}}+1\right)^{2}}-\sqrt{\left(\frac{\varepsilon}{\varepsilon_{y}}-1\right)^{2}}\right)-f_{c^{\prime}} \cdot\left[k_{1} \varepsilon+k_{2} \varepsilon^{2}+k_{3} \varepsilon^{3}+k_{4} \varepsilon^{4}\right]$

Cada uno de los puntos de los diagramas se ha calculado mediante la imposición a priori de cuatro condiciones:

- Se ha fijado el rumbo del eje neutro.

- Se ha impuesto que el agotamiento de la sección se alcanza por compresión en el hormigón en la fibra extrema, lo cual implica dotar de ductilidad ilimitada al acero. Desde un punto de vista operativo es preciso acotar el valor de deformación para describir la ecuación constitutiva, y este se ha fijado en $\varepsilon_{y u}=0.020$, (figura 5). Se verifica que en ningún plano de agotamiento determinado se alcanza dicho valor. Este modelo de agotamiento es el empleado por la ACI-318.

- No se han empleado coeficientes de minoración en los materiales.

- No se han empleado coeficientes de minoración de la capacidad de la sección.

La variable libre del plano de agotamiento que ha de ser determinada para cada punto del diagrama es la profundidad del eje neutro. Su obtención se ha llevado a cabo de manera iterativa. El proceso de búsqueda de cada profundidad cesa cuando el axil interno logrado (luego de haber resuelto la ecuación [11] para el plano postulado) es igual al exterior. En este análisis, se ha considerado válida una profundidad de cada eje neutro cuando la diferencia entre axiles (buscado y calculado) es inferior al 0.01\%, [15].

$$
P_{d}-P \leq 0.0001 P
$$

La figura 6 muestra los 24 diagramas de interacción calculados.
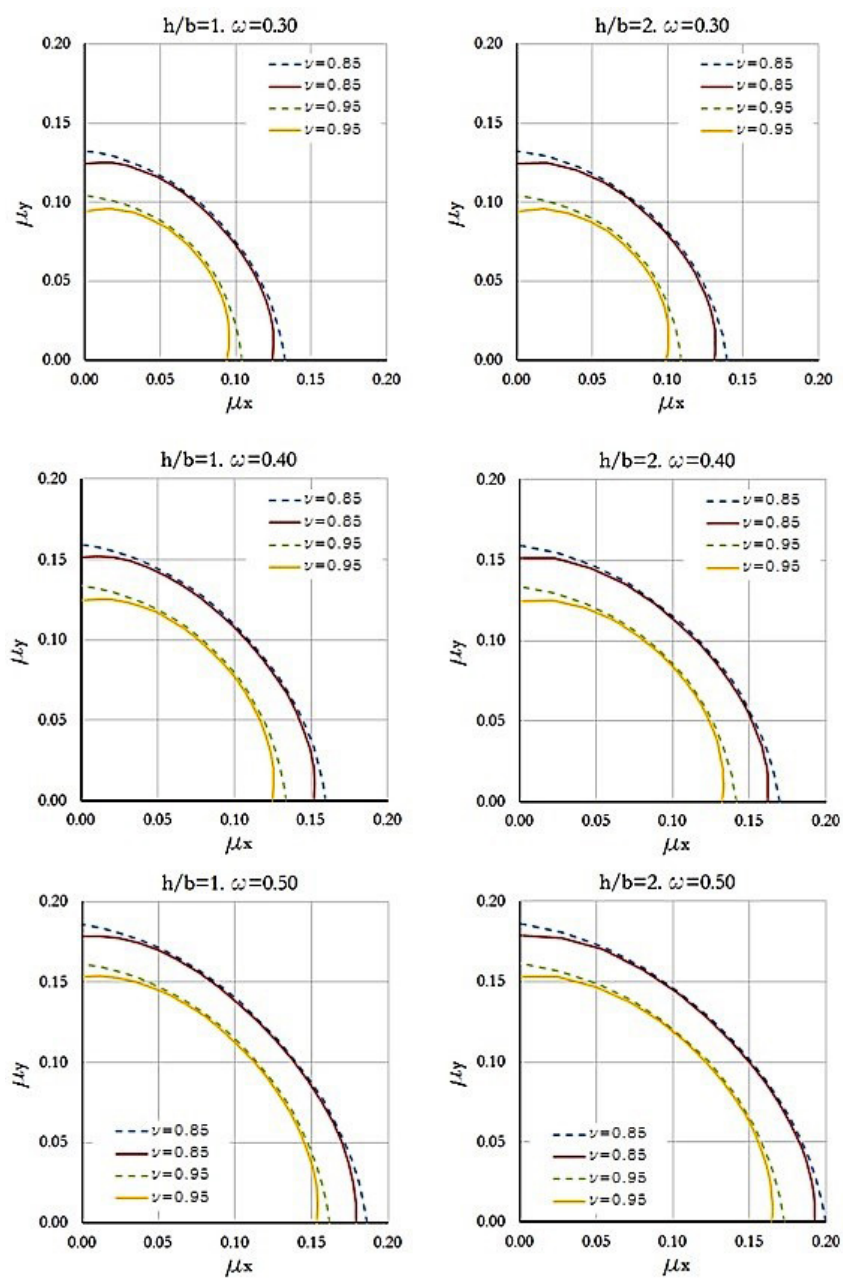

Figura 6. Diagramas de interacción determinados para las secciones descritas en la tabla 1. Línea continua denota modelo hiperbólico, línea discontinua denota modelo parábola-rectángulo.

Para los 24 casos analizados se ha determinado el valor máximo de la resultante del momento resistente de la sección como composición vectorial de las componentes referidas a los ejes de simetría según la expresión [16] (en términos adimensionales), así como el giro para el que se ha encontrado dicho valor máximo. Como cabía esperar para todas las secciones en las que se ha empleado un modelo constitutivo parábola-rectángulo, el valor de resistencia máximo se ha encontrado para el eje de simetría de mayor inercia, eje $x$.

$$
M=\sqrt{M_{x}^{2}+M_{y}^{2}}
$$

\section{DISCUSIÓN DE LOS RESULTADOS OBTENIDOS}

En la tabla 1 se muestran los valores significativos de los diagramas calculados cuyos significados se detallan seguidamente:

$\frac{h}{b}$ : Relación entre el canto y la base de la sección transversal. $\omega$ : Cuantía mecánica, descrita en [7].

Modelo Hormigón: tipo de diagrama tensión deformación empleado.

$v$ : Axil característico reducido, descrito en [8].

$\mu\left(\alpha=0^{\circ}\right)$ : Resultante reducida del vector momento resistente referido al eje $\mathrm{x}$ de la sección. 
$\mu\left(\alpha=90^{\circ}\right)$ : Resultante reducida del vector momento resistente referido al eje y de la sección.

Ø: Ángulo de resistencia máxima de la sección referido al eje principal de simetría de la sección.

$\mu(\alpha=\varnothing)$ : Resultante reducida del vector momento resistente para la dirección de resistencia máxima de la sección (Ø).

$\Delta \mu_{x}(\%)$ : diferencia en porcentaje entre las resultantes de momentos resistentes referidos al eje $\mathrm{x}$ de dos secciones idénticas para los dos modelos constitutivos analizados.

Como puede apreciarse en los valores de la tabla 1, para las secciones en las que se ha empleado un modelo constitutivo hiperbólico en el hormigón, el valor de resistencia máximo de la sección no coincide con la dirección del eje principal de simetría, y en todos los casos el valor de la resultante de momento resistente referido al eje de simetría principal (eje $x$ ) es inferior al valor de la resultante de momento resistente según dicha dirección para un modelo constitutivo tipo parábola-rectángulo.

Este hecho se produce debido a que en situaciones de axiles elevados, empleando un modelo hiperbólico, la tensión máxima en la sección transversal no coincide con la fibra extrema ya que en esa posición ha de lograrse la deformación última $\varepsilon_{c u}$, y esto provoca que el baricentro de la resultante del bloque comprimido de hormigón se aproxime a la posición de la fibra neutra reduciéndose el brazo mecánico y provocando que el momento resultante se reduzca. En la figura 7 se muestra el perfil de tensiones en el hormigón sobre la sección analizada $\frac{h}{b}=1, \omega=0.30, v=0.95$, para los dos modelos constitutivos empleados, y 500x500 $\mathrm{mm}^{2}$ de dimensiones, situación en la que se ha obtenido la mayor diferencia entre los momentos resistentes de la sección referidos a su eje principal de simetría (9.615\%). Es posible apreciar como el brazo mecánico de la sección en la figura $7 \mathrm{~b}$ es menor que en la figura 7a. También se observa como en la zona de máxima deformación (fibra superior de la figura $7 \mathrm{~b}$ ), el perfil de tensiones disminuye de valor, haciendo compatible la tensión según el diagrama tensión deformación hiperbólico mostrado en la figura 4b.

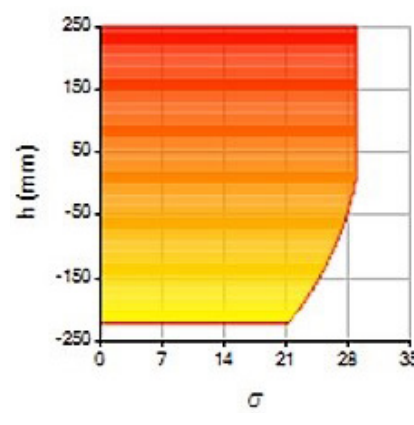

(a)

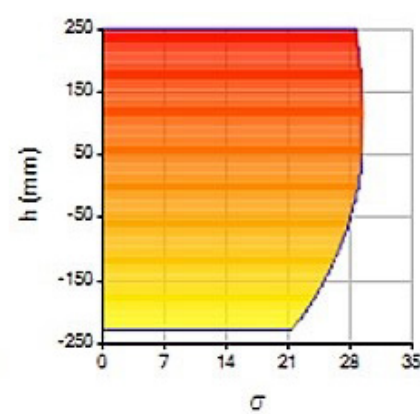

(b)
Figura 7. Perfil de tensiones de la sección analizada $\frac{\mathrm{h}}{\mathrm{b}}=1$, $\omega=0.30, v=0.95$, para los dos modelos constitutivos empleados, y $500 x 500 \mathrm{~mm}^{2}$ de dimensiones analizada según los dos modelos constitutivos descritos. (a) Ecuación parábola-rectángulo. (b), Ecuación hiperbólica.

Tabla 1. Valores significativos calculados para los 24 casos analizados.

\begin{tabular}{|c|c|c|c|c|c|c|c|c|}
\hline$\frac{\boldsymbol{h}}{\boldsymbol{b}}$ & $\boldsymbol{\omega}$ & $\begin{array}{l}\text { Modelo } \\
\text { Hormigón }\end{array}$ & $\boldsymbol{v}$ & $\mu\left(\alpha=0^{\circ}\right)$ & $\mu\left(\alpha=90^{\circ}\right)$ & $\varnothing\left(^{\circ}\right)$ & $\mu(\alpha=\varnothing)$ & $\Delta \mu_{x}(\%)$ \\
\hline 1 & 0.30 & Parabólico & 0.85 & 0.133 & 0.133 & 0.00 & 0.133 & \multirow{2}{*}{6.767} \\
\hline 1 & 0.30 & Hiperbólico & 0.85 & 0.124 & 0.124 & 13.00 & 0.126 & \\
\hline 1 & 0.30 & Parabólico & 0.95 & 0.104 & 0.104 & 0.00 & 0.104 & \multirow{2}{*}{9.615} \\
\hline 1 & 0.30 & Hiperbólico & 0.95 & 0.094 & 0.094 & 40.00 & 0.100 & \\
\hline 1 & 0.40 & Parabólico & 0.85 & 0.159 & 0.159 & 0.00 & 0.159 & \multirow{2}{*}{4.403} \\
\hline 1 & 0.40 & Hiperbólico & 0.85 & 0.152 & 0.152 & 10.00 & 0.153 & \\
\hline 1 & 0.40 & Parabólico & 0.95 & 0.134 & 0.134 & 0.00 & 0.134 & \multirow{2}{*}{6.716} \\
\hline 1 & 0.40 & Hiperbólico & 0.95 & 0.125 & 0.125 & 16.50 & 0.127 & \\
\hline 1 & 0.50 & Parabólico & 0.85 & 0.186 & 0.186 & 0.00 & 0.186 & \multirow{2}{*}{3.763} \\
\hline 1 & 0.50 & Hiperbólico & 0.85 & 0.179 & 0.179 & 3.00 & 0.179 & \\
\hline 1 & 0.50 & Parabólico & 0.95 & 0.161 & 0.161 & 0.00 & 0.161 & \multirow{2}{*}{4.969} \\
\hline 1 & 0.50 & Hiperbólico & 0.95 & 0.153 & 0.153 & 8.50 & 0.155 & \\
\hline 2 & 0.30 & Parabólico & 0.85 & 0.139 & 0.133 & 0.00 & 0.139 & \multirow{2}{*}{$5 \cdot 755$} \\
\hline 2 & 0.30 & Hiperbólico & 0.85 & 0.131 & 0.124 & 19.00 & 0.133 & \\
\hline 2 & 0.30 & Parabólico & 0.95 & 0.109 & 0.104 & 0.00 & 0.109 & \multirow{2}{*}{9.174} \\
\hline 2 & 0.30 & Hiperbólico & 0.95 & 0.099 & 0.094 & 50.00 & 0.104 & \\
\hline 2 & 0.40 & Parabólico & 0.85 & 0.170 & 0.159 & 0.00 & 0.170 & \multirow{2}{*}{4.706} \\
\hline 2 & 0.40 & Hiperbólico & 0.85 & 0.162 & 0.152 & 13.50 & 0.163 & \\
\hline 2 & 0.40 & Parabólico & 0.95 & 0.141 & 0.134 & 0.00 & 0.141 & \multirow{2}{*}{6.383} \\
\hline 2 & 0.40 & Hiperbólico & 0.95 & 0.132 & 0.125 & 21.50 & 0.134 & \\
\hline 2 & 0.50 & Parabólico & 0.85 & 0.200 & 0.186 & 0.00 & 0.200 & \multirow{2}{*}{4.000} \\
\hline 2 & 0.50 & Hiperbólico & 0.85 & 0.192 & 0.179 & 10.00 & 0.193 & \\
\hline 2 & 0.50 & Parabólico & 0.95 & 0.173 & 0.161 & 0.00 & 0.173 & \multirow{2}{*}{5.202} \\
\hline 2 & 0.50 & Hiperbólico & 0.95 & 0.164 & 0.153 & 16.50 & 0.165 & \\
\hline
\end{tabular}




\section{CONCLUSIONES}

A la luz de los resultados obtenidos, es posible realizar las siguientes afirmaciones:

- En secciones de hormigón armado con dos ejes de simetría no es posible afirmar que el diagrama de interacción de agotamiento sea convexo en toda su dominio, y esta afirmación es cierta al menos en situaciones en las que se empleen modelos constitutivos de hormigón en los que la deformación en rotura tenga asociados valores de tensión inferiores a los máximos.

- El eje principal de simetría de una sección rectangular de hormigón armado no tienen necesariamente por qué ser el eje de mayor inercia de la sección, y como consecuencia de este hecho, el valor resistente máximo de la sección no necesariamente se encuentra según la dirección esperada (eje $x$ ).

- El giro de desvío entre el eje de máxima resistencia y el eje principal de simetría de una sección de hormigón armado doblemente simétrica aumenta al tiempo que se incremente el axil.

- La diferencia de capacidad de la sección a flexión compuesta respecto al eje principal de simetría de la sección para los modelos parábola-rectángulo e hiperbólico aumenta al tiempo que disminuye la cuantía de armadura. Expresado en otros términos es posible afirmar que para cuantías bajas, la reducción de la capacidad de la sección en flexión compuesta recta respecto al eje principal de simetría de la sección aumenta.

- El giro de desvío entre el eje de resistencia máxima y el eje principal de simetría de hormigón armado doblemente simétrica, aumenta al tiempo que la diferencia entre la tensión máxima del hormigón en compresión y la tensión para la deformación de agotamiento se incrementa.

- El empleo de modelos simplificados para la caracterización del hormigón en compresión conduce, en secciones doblemente simétricas de hormigón armado y axiles elevados, a otorgar una resistencia mayor a la real a la sección frente a esfuerzos de flexión compuesta recta.

- No se descarta la hipótesis de convexidad en diagramas de interacción de secciones de hormigón armado con doble simetría para modelos constitutivos de hormigón en los que la tensión máxima se produzca para la deformación de agotamiento. Expresado en otros términos no es posible afirmar que los ejes principales de inercia no coincidan con los ejes de simetría de una sección cuando se emplean modelos constitutivos para la descripción del hormigón del tipo parábola-rectángulo o rectangulares (hipótesis de Withney).

\section{LISTA DE ABREVIATURAS}

b: Base de la sección trasversal.

$f_{u}$ : Tensión normal de agotamiento en el acero.

$f_{c}$ : Tensión normal de agotamiento en el hormigón.

h: Canto de la sección transversal.

$k_{i}$ : Coeficientes en la descripción de la ecuación constitutiva de hormigón.

r: Recubrimiento mecánico de la armadura de la sección transversal.

A: Área de la sección transversal.

$M$ : Momento flector resultante de la composición vectorial de sus componentes.

$M_{x}$ : Momento flector referido al eje $x$ de la sección.

$M_{y}$ : Momento flector referido al eje $y$ de la sección.

$\Delta M_{x}$ : Diferencia de la componente referida al eje $x$ de la sección del momento flector.

$P$ : Axil.

$P_{0}$ : Axil de agotamiento aplicado en el centro de gravedad de la sección transversal.

$P_{d}$ : Axil de diseño exterior considerado.

$P_{i}$ : Axil de agotamiento aplicado en un punto $i(x, y)$.

$P_{x}$ : Axil de agotamiento aplicado en un punto $i(x, 0)$.

$P_{y}$ : Axil de agotamiento aplicado en un punto $i(0, y)$.

$\alpha$ : Ángulo de la fibra neutra.

$\varepsilon$ : Deformación unitaria.

$\varepsilon_{c 0}$ : Deformación a la que se alcanza la tensión de agotamiento en el hormigón.

$\varepsilon_{c u}$ : Deformación a la que se alcanza el agotamiento en el hormigón.

$\varepsilon_{y}$ : Deformación en el límite elástico en el acero.

$\varepsilon_{y u}$ : Deformación en la que se alcanza el agotamiento en el acero.

$\mu$ : Momento flector reducido.

$v$ : Axil reducido.

$\omega$ : Cuantía de armadura de la sección transversal.

$\sigma_{c}$ : Tensión normal en un elemento de hormigón.

$\sigma_{s}$ : Tensión normal en un elemento de acero.

Ø: Ángulo de resistencia máxima de la sección referido al eje principal de simetría de la sección.

\section{REFERENCIAS}

(1) Ministerio de Fomento, Comisión Permanente del Hormigón (2008). Instrucción de Hormigón Estructural EHE-o8.

(2) Withney, C., Edward, C., (1956). Guide for Ultimate Strength Design of Reinforced Concrete. ACI Journal, Proceedings V.53, 445-490.

(3) Hognestad, E. (1951). A Study of Combined Bending and Axial Load in Reinforced Concrete Members. Bulletin $\mathrm{N}^{\circ} 399$, Engineering Experimental Station, University of Illinois, Urbana, $128 \mathrm{pp}$

(4) Kent, D. C., Park, R. (1971). Flexural Members with Confined Concrete. Journal of the Structural Division, American Society of Civil Engineers, 7, pp. 1969-1990.

(5) Sargin, M. (1971). Stress-strain Relationship for Concrete and the Analysis of Structural Concrete Sections. Solid Mechanics Division, University of Waterloo, Waterloo, Ontario.

(6) Morán, F. (1972). Design of reinforced concrete sections under normal loads and stresses in the ultimate state. CEB Bulletin d'Information $\mathrm{N}^{0} 83$

(7) Pannell, F.N. 1963. "Failure surfaces for members in compression and biaxial bending." ACI Journal, Proceedings, 60 (1): 129-140.

(8) Bresler, B. (1960). Design criteria for reinforced columns under axial load and biaxial bending. ACI Journal, Proceedings, 57, 484-490.

(9) ACI Committee 318, American Concrete Institute. (2014). Building Code Requirements for Structural Concrete ACI-318-14 
(10) Parme, A. L, Nieves, J. M., Gouwens, A. (1966). Capacity of Reinforced Rectangular Columns Subject to Biaxial Bending. ACI Journal, Proceedings, 63, 911-923.

(11) Weber, D. C. (1966). Ultimate Strength Design Charts for Columns with Biaxial Bending. ACI Journal, Proceedings, 63, 1205-1320, Disc., 1538-1586.

(12) Paulay T., Row D. G. (1973). Biaxial flexure and axial load interaction in short rectangular reinforced concrete columns. Bulletin of New Zealand Society for Earthquake Engineering.

(13) Grasser E. (1981). Manual CEB/FIP on Bending and Compression, Bulletin D’ Information n.$^{\circ} 141$, Construction Press.

(14) Papanikolaou V. K. (2012). Analysis of arbitrary composite sections in biaxial bending and axial load. Computers and Structures 98-99:33-54.

(15) Lejano B. A. (2007). Investigation of Biaxial Bending of Reinforced Concrete Columns Through Fiber Method Modeling. Journal of Research in Science, Computing, and Engineering 4:3, 61-73.

(16) Gil-Martín L.M., Hernández-Montes E., Aschheim M. (2007). Optimal reinforcement of RC columns for biaxial bending. Material and Structures 43(9):1245-56.

(17) Leite L. C, Bonet S. J, Migel S. P., Pallarés R. L. (2012). Estudio experimental de soportes de hormigón armado sometidos a flexo-compresión con excentricidades y ángulos de esviaje desiguales en los extremos. Encontro Nacional Betau Estrutural - BE2012 FEUP.

(18) Sánchez-Olivares, G., Tomás, A. (2017). Improvements in meta-heuristic algorithms for minimum cost design of reinforced concrete rectangular sections under compression and biaxial bending. Engineering Structures, 130, pp 162-179.

(19) Farah, A., Huggins, M. W. (1969). Analysis of Reinforced Concrete Columns Subjected to Longitudinal Load and Biaxial Bending. ACI Journal, Proceedings, 66. 569-575. 